\title{
Characterization of single lesion nonfunctioning pancreatic neuroendocrine carcinoma via computed tomography
}

\author{
JIBO HU, QUNHUI HU and HONGJIE HU \\ Department of Radiology, Sir Run Run Shaw Hospital, \\ Zhejiang University School of Medicine, Hangzhou, Zhejiang 310016, P.R. China
}

Received October 23, 2015; Accepted October 24, 2016

DOI: $10.3892 / 01.2017 .5729$

\begin{abstract}
Nonfunctioning pancreatic neuroendocrine carcinoma (NPNEC) is a poorly differentiated neuroendocrine carcinoma and a rare type of pancreatic cancer. Computed tomography (CT)-based imaging is currently the most effective method for diagnosing and classifying NPNEC. The present study investigated the clinical features and CT manifestations of this disease. The CT manifestations and clinical data of 13 patients with NPNEC, confirmed by surgery or needle biopsy, were retrospectively analyzed. None of the 13 patients exhibited endocrine-related symptoms and a single lesion with a variable position in the pancreatic was observed in all patients. The tumors were generally round or irregular, with inhomogeneous density and an average diameter of $7.45 \pm 3.92 \mathrm{~cm}$ (range, 4.0-16.0 cm). Cystic necrosis zones were observed in 5 patients and calcification was observed in 2 . A total of 4 patients exhibited intrahepatic metastases, and 2 patients demonstrated spleen and colon invasion. Enhanced scanning indicated that tumors were markedly enhanced. There were 11 cases of middle or high-grade enhancement (84.6\%) and 2 cases of mild enhancement (15.4\%). Marked envelope enhancement was observed in $3 / 11$ patients with high-grade enhancement. In conclusion, the majority of patients with NPNEC lacked characteristic manifestations. While the CT examination revealed certain characteristics, histological analysis was necessary to confirm diagnoses.
\end{abstract}

\section{Introduction}

Pancreatic neuroendocrine carcinoma (PNEC), otherwise known as islet cell tumors, is a diverse group of rare neoplasms originating from endocrine or nerve cells within the pancreas (1), and accounts for $1-2 \%$ of all pancreatic tumors (2,3). PNEC may be classified as either functioning

Correspondence to: Dr Jibo Hu, Department of Radiology, Sir Run Run Shaw Hospital, Zhejiang University School of Medicine, 3 East Qingchun Road, Hangzhou, Zhejiang 310016, P.R. China E-mail: jiebohu201510@sina.com

Key words: nonfunctioning pancreatic neuroendocrine carcinoma, clinical features, computed tomography manifestation pancreatic neuroendocrine carcinoma or nonfunctioning pancreatic neuroendocrine carcinoma (NPNEC) depending on the production levels of specific pancreatic endocrine hormones, including gastrin, insulin and glucagon, and the relevant associated clinical symptoms such as insulinoma, gastrinoma, VIPoma, glucagonoma, and somatostatinoma due to hormonal hypersecretion $(1,3,4)$.

Neuroendocrine neoplasms are a series of heterogeneous tumors derived from peptidergic neurons or neuroendocrine cells, and their clinical presentations range from low-grade malignancies with slow growth to marked malignancies with a high level of metastasis based on the degrees of differentiation (5). NPNEC is a poorly-differentiated neuroendocrine carcinoma and is rare, representing $<2 \%$ of all pancreatic cancer cases $(1,6)$. The median overall disease-related survival of patients with NPNEC is 9.7 years (7), thus confirming the generally long-term survival of patients with this disease, and the 5-year survival rate ranges from 50-65\% (8-11). Radical surgery is the most effective therapeutic strategy for NPNEC and the 5-year survival rate of patients who have undergone surgery is $93 \%(7,12)$. However, the decision to refer a patient for surgery is dependent on the disease stage at diagnosis (7). Therefore, accurate diagnosis of NPNEC is particularly important to achieve a more positive outcome.

Computed tomography (CT) is currently the optimal method for diagnosing and staging suspected cases of pancreatic carcinoma (13). Moreover, it has been demonstrated that particular risk factors including age (13), smoking (14), diabetes and obesity may contribute to the development of pancreatic carcinoma (15). The diagnosis of NPNEC has major differences compared to those of pancreatic cancer derived from exocrine tissues (16), which accounts for $>85 \%$ of pancreatic carcinoma. Therefore, summarizing the characterizations of CT-based NPNEC diagnosis based on the analysis of CT scans is necessary. The present study retrospectively analyzed the clinical data and CT manifestations of 13 patients with NPNEC confirmed by surgery or needle biopsy. A literature review of the clinical features, CT manifestations and CT diagnostic values of NPNEC was also conducted.

\section{Patients and methods}

Patient clinicopathological factors. A total of 13 patients admitted to Department of Radiology, Sir Run Run Shaw 
Hospital, Zhejiang University School of Medicine (Hangzhou, China) were enrolled in the present study, including 7 men and 6 women, and the mean patient age was $46.4 \pm 9.2$ years (range, 38-60 years). All 13 patients exhibited a single lesion inside the pancreas and 4 patients also had combined multiple intrahepatic metastases. A total of 2 patients had spleen and colon invasion, and of these, 1 patient also exhibited left kidney involvement. All patients underwent surgery or CT-guided needle biopsy between July 2012 and October 2014 in the Sir Run Run Shaw Hospital, in addition to routine pathology and immunohistochemistry examination. The diagnoses of all patients were all confirmed as NPNEC.

Institutional Ethics Board approval was obtained from the Medical Ethics Committee of Sir Run Run Shaw Hospital, Zhejiang University School of Medicine (Hangzhou, China). All participating patients were formally informed of the purpose of the study and written informed consent was obtained from all participants.

Examination methods. CT examinations were performed using GE Hi Speed NX/I Dual Slice CT Scanner and Siemens SOMATOM Sensation 16 CT scanners. The three-phase scanning method (17) was used with a non-enhanced scan and an enhanced scan performed at the arterial and venous phases. The arterial and venous phases began 25 and $65 \mathrm{sec}$, respectively, following injection of the contrast agent. The scan thickness was $7 \mathrm{~mm}$, scan spacing was $7 \mathrm{~mm}$ and the pitch was 1.0. Iohexol (GE healthcare China, Shanghai, China) and ioverol (HengRui Pharmacy, Jiangsu, China) were used as contrast agents. The iohexol was used for 5 patients while ioverol was used for 8 patients depending on the availability of agent when patient enrolled The injection rate was $2.8-3.0 \mathrm{ml} / \mathrm{s}$, and the dose was 90-100 $\mathrm{ml}$.

Hemotoxylin and eosin staining and immunohistochemistry. For histochemical analysis, the collected samples were fixed in $10 \%$ formaldehyde for $24 \mathrm{~h}$ at $4^{\circ} \mathrm{C}$ and samples from patients were mounted onto slides ( $4 \mu \mathrm{m}$-thick) embedded with paraffin and stained with hematoxylin and eosin. For immunohistochemistry, paraffin sections were subsequently deparaffinized with xylene and rehydrated in decreasing concentrations of ethanol. Antigen retrieval was performed by heating sections in an antigen retrieval buffer ( $\mathrm{pH}$ 6.0) in a microwave cooker at $90^{\circ} \mathrm{C}$ for $45 \mathrm{~min}$. Endogenous peroxidase activity was blocked with $3 \% \mathrm{H}_{2} \mathrm{O}_{2}$ for $10 \mathrm{~min}$, followed by the blocking of endogenous biotin enzyme with normal goat serum (Sigma-Aldrich, St. Louis, MO) for $30 \mathrm{~min}$ at room temperature. Sections were then incubated with a 1:40 dilution (same dilution applied for all primary antibodies) of rabbit anti-chromogranin A antibody (cat. no., SAB4200668-100UL; Sigma-Aldrich; Merck Millipore, Darmstadt, Germany), anti-cytokeratin antibody (cat. no., C2562-100UL; Sigma-Aldrich; Merck Millipore) and anti-synaptophysin antibody (cat. no., S5768-2ML; Sigma-Aldrich; Merck Millipore) overnight at $4^{\circ} \mathrm{C}$, washed with PBS and incubated with anti-mouse (cat. no., A9044-2ML; Sigma-Aldrich; Merck Millipore) or anti-rabbit (cat. no., RABHRP1-10UL; Sigma-Aldrich; Merck Millipore) horseradish peroxidase-conjugated antibodies (1:100 dilution for both) at room temperature for $60 \mathrm{~min}$. Sections were subsequently washed in PBS. Immunostaining was visualized in Olympus CX22 microscope using diaminobenzidine chromogen and the sections were counterstained with hematoxylin. Two pathologists blinded to patient's information separately evaluated all slides. Brown staining in cytoplasm was defined as positive staining. The scoring system used incorporated the intensity of the staining (absent, weak, moderate or strong) and the percentage of positively stained tumor cells. A positive sample was defined as a sample with $>5 \%$ of the tumors cells demonstrating moderate or strong staining. Weak positive samples were those where $>5 \%$ of the tumors cells demonstrated weak staining. Extremely weak positive samples were defined as samples where $<5 \%$ of the tumor cells demonstrated weak staining.

\section{Results}

Clinical manifestation features. None of the 13 patients demonstrated endocrine-related clinical symptoms. A total of 10 patients were admitted due to palpable masses in the upper abdomen, of which 5 patients had combined non-specific digestive system symptoms, including upper abdominal pain, discomfort and loss of appetite, and 2/10 patients experienced combined emaciation and weight loss. A further 2 patients were admitted due to pancreatic masses identified following during physical examinations and 1 patient was admitted due to a pancreatic mass identified following a thoracic injury. Laboratory examination results indicated that 1 patient experienced a slight increase in levels of carbohydrate antigen 19-9, 2 patients were weakly positive for glucagon, 3 patients were extremely weakly positive for glucagon, 1 patient was extremely weakly positive for somatostatin, 2 patients were weakly positive for insulin and 4 patients were weakly positive and extremely weakly positive for multiple hormones, such as insulin, glucagon, gastrin and vasoactive intestinal peptide.

Tumor characterization. All patients enrolled in the study presented with one single space-occupying lesion in the pancreas, of which 7 cases were in the pancreatic head, 1 was in the pancreatic body, 1 was in the tail of the body and 4 were in the pancreatic tail. The lesions were generally round or irregular, and the average maximum tumor diameter was $7.45 \pm 3.92 \mathrm{~cm}$ (range, $\sim 4.0-16.0 \mathrm{~cm}$ ).

CT manifestations. Non-enhanced CT scans of the tumors demonstrated homogeneous or inhomogeneous consistency with a slightly low density. Necrotic cystic areas were observed in the tumors of 5 patients, and 2 patients had striped and speckled calcification within the tumors. A total of 7 patients exhibited a vague border between the tumor and the normal pancreas, while the other 6 patients exhibited a clear border between the tumor and the normal pancreas. Following enhanced scanning, marked enhancement was observed in the tumors of 5 patients, moderate enhancement in 6 patients and mild enhancement in 2 patients. The enhancement peak values were in the arterial phase for 5 patients and in the parenchymal phase for 8 patients. In the solid section, 9 patients exhibited homogeneous enhancement, whereas 4 patients exhibited inhomogeneous enhancement. No enhancement was observed in necrotic cystic areas. Tumor envelope enhancement was 

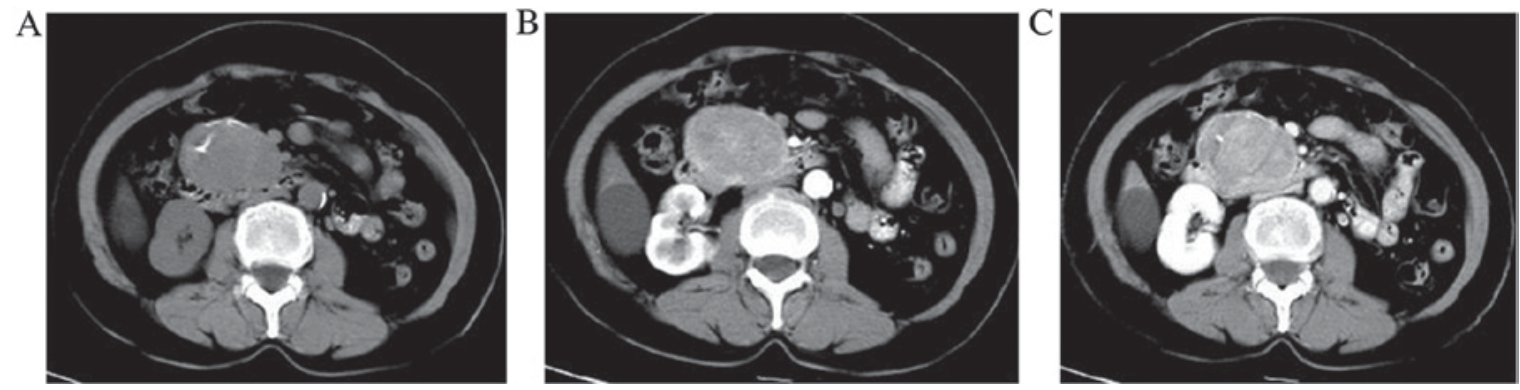

Figure 1. Representative images of nonfunctioning pancreatic neuroendocrine carcinoma in the pancreatic head. (A) Plain CT scanning indicated a slightly low density space-occupying tumor $(4.5 \times 6.8 \mathrm{~cm})$ in the pancreatic head. The border was clear, and speckled calcification was observed within the tumor. Enhanced scanning of the tumor demonstrated moderate, gradual and homogeneous enhancement. The envelope exhibited obvious enhancement. (B) Arterial phase enhanced CT scan and (C) parenchymal phase enhanced CT scan. CT, computed tomography.

observed in 3 patients and the enhancement degree was more obvious than that of the tumor entity (Figs. 1 and 2).

Clinical syndromes. In total, 2 patients exhibited dilated biliary ducts and 1 patient exhibited a dilated pancreatic duct; the dilated pancreaticobiliary ducts were homogeneous and the degrees were mild. A total of 4 patients exhibited combined multiple intrahepatic metastases; there were 3 cases of cystic metastatic lesions and 1 case of a solid metastatic lesion. The enhancement features were similar to those of primary pancreatic cancer. Furthermore, 2 patients exhibited spleen and colon invasion, of which 1 patient had left kidney involvement. An additional patient exhibited hilar lymph node enlargement, 2 patients had peritumoral blood vessel involvement (primarily pressing shift and tortuous expansion changes; 1 patient had a filling defect in the splenic blood vessels) and spleen enlargement was observed in 2 patients (Fig. 3).

Pathological examination. All 13 patients were diagnosed with NPNEC. Immunohistochemistry results demonstrated that 11 patients were positive for chromogranin A, 8 for synaptophysin, 8 for cytokeratin and 1 was weakly positive for protein gene product 9.5 . Representative immunohistochemistry images are presented in Fig. 4.

\section{Discussion}

NPNEC primarily occurs in middle-aged and young patients, with no marked gender difference (18). In the current study, 7 patients were men (54\%) and 6 were women (46\%), and the average patient age was $46.4 \pm 9.2$ years, which is higher than the average age of patients with NPNEC at diagnosis. NPNEC usually grows slowly, and as it does not exhibit specific clinical manifestation, it is often misdiagnosed as a digestive system disease, such as gastritis. The tumors are typically large and demonstrate clear space-occupying effects at the time of identification. A previous study indicated that when NPNEN tumors were identified, their volume was usually large $(>3 \mathrm{~cm})(19)$. In the current study, the maximum tumor diameter was $>4 \mathrm{~cm}$, and average diameter was $7.45 \pm 3.92 \mathrm{~cm}$, which is similar to that reported in a previous study $(15,19)$.

NPNEC tumors may occur at any location in the pancreas; however, they most commonly occur in the pancreatic head $(16,18)$. In the present study, the lesions of 7 patients were located in the pancreatic head. The presentations of tumors in the plain CT scans were generally homogeneous or inhomogeneous with a slightly low density or isodensity. A total of 11 patients in the present study exhibited at least moderate enhancement, whereas 2 patients exhibited mild enhancement, which was speculated to be associated with more fibrous components in the tumors. Enhancement peaks may be observed at the arterial and parenchymal phase, and there was no clear specificity for a single case. Certain studies consider envelope enhancement as a characteristic presentation of NPNEC (19), yet only 3 patients in the present study exhibited this feature. Morphologically, tumor cells exhibit poor differentiation and increased volume compared with normal cells, and liquefaction necrosis is generally observed at the center of tumors (20). A total of 5 patients in the current study exhibited signs of necrotic cystic lesions. Noone et al (21) reported that $\sim 20 \%$ of NPNEC tumors exhibit calcifications, which are isolated and nodular in shape, and noted that the incidence of calcification is higher than that observed in pancreatic cancer, which is $2 \%$.

Peripancreatic lymph node enlargement and hepatic metastasis are two important features of NPNEC. It has been proposed that the major metastatic site of PNEC is the local lymph nodes $(18,22)$. Noone et al (21) suggested that the presentation of NPNEC was similar to that of pancreatic cancer; malignant lymph nodes or distant metastasis were observed during surgery in $83 \%$ patients and fatal bleeding was reported in $\geq 20 \%$. One possible reason for the bleeding may have been tumor rupture or invasion of large blood vessels by tumors. In the current study, 4 patients experienced distant hepatic metastasis, 1 patient exhibited hilar lymph node metastasis, and compression or invasion of peritumoral blood vessels was observed in 2 patients; however, no cases experienced massive bleeding. It was therefore suggested that the probability of hepatic metastasis in NPNEC patients diagnosed by CT was higher than that of local lymph node metastasis.

Due to compression by tumors, patients with NPNEC may exhibit combined pancreaticobiliary duct dilation, whereby the edge of the dilated pancreaticobiliary duct is smooth and the thickness is more homogeneous. These patients may also exhibit peritumoral blood vessel involvement and splenomegaly. In the present study, combined biliary duct dilation was observed in 2 patients and 1 patient exhibited pancreatic duct dilation; the degrees were all mild. Spleen enlargement 

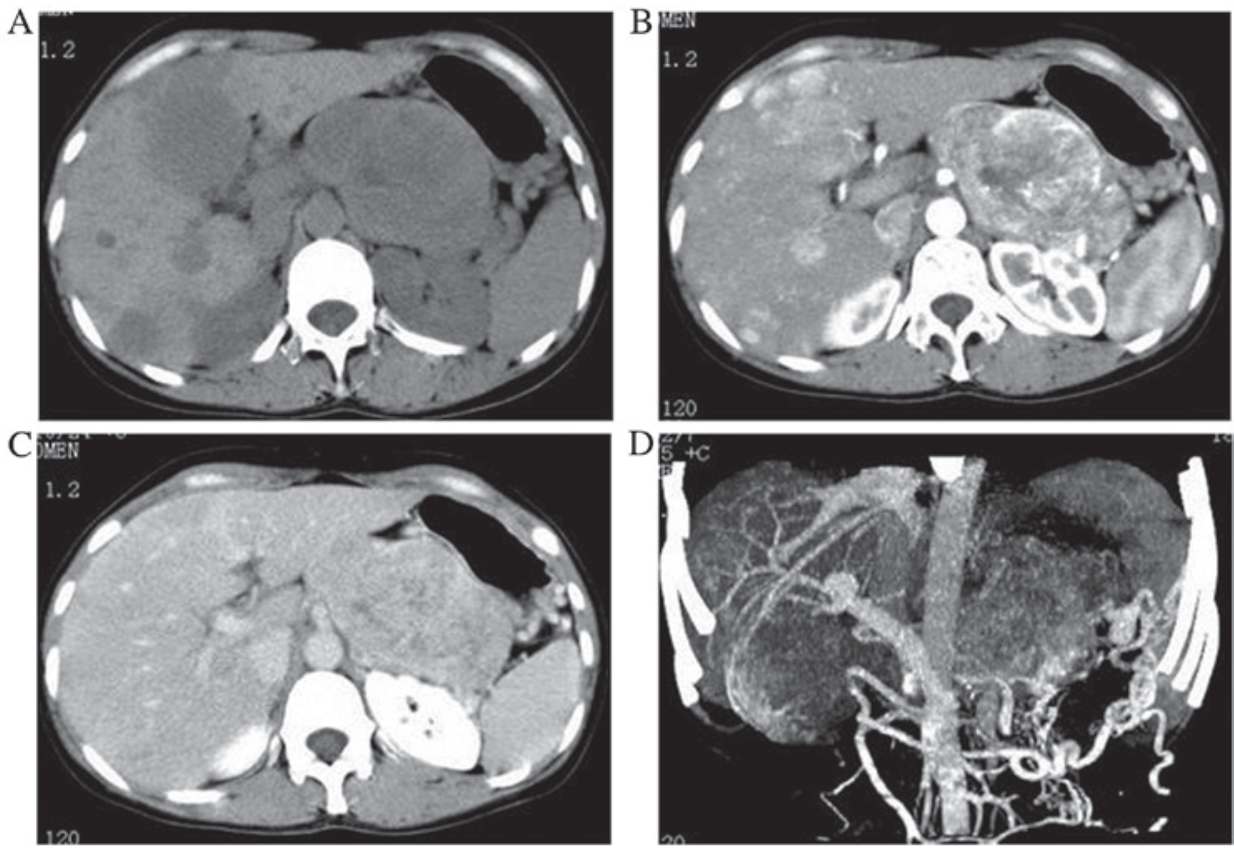

Figure 2. Representative images of nonfunctioning pancreatic neuroendocrine carcinoma in the tail of the pancreatic body. (A) Plain CT scanning indicated a slightly low density space-occupying tumor $(6.3 \times 9.5 \mathrm{~cm})$ in the tail of the pancreatic body; the density was inhomogeneous. (B) Enhanced scanning indicated marked tumor enhancement, obvious enhancement of the envelope and tortuous expansion of the blood vessels surrounding the tumor. (C) Parenchymal phase enhanced CT scan and (D) CT multiplanar reconstruction; there were multiple intrahepatic metastatic lesions and the enhancement style was the same as that in the primary pancreatic tumor. CT, computed tomography.
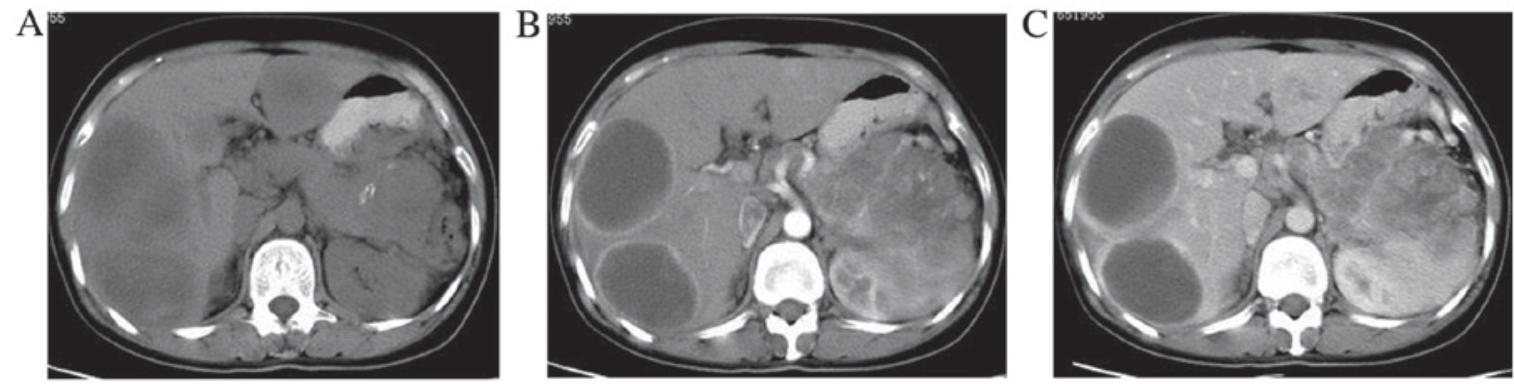

Figure 3. Representative images of nonfunctioning pancreatic neuroendocrine carcinoma in the tail of the pancreatic body. (A) Plain CT scanning revealed a slightly low-density space-occupying tumor with a maximum diameter of $\sim 16 \mathrm{~cm}$ in the tail of the pancreatic body. Striped calcification was present inside the tumor. (B) Enhanced scanning of the tumor indicated marked inhomogeneous enhancement. The edge was blurry. The tumor had invaded the spleen, colon and left kidney, and splenic artery stenosis was present. There were multiple intrahepatic cystic metastatic lesions. (C) Parenchymal phase enhanced CT scan. $\mathrm{CT}$, computed tomography.

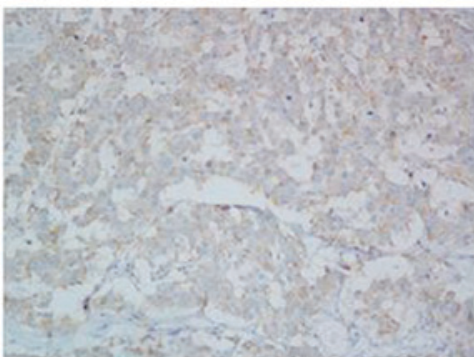

Anti-CgA

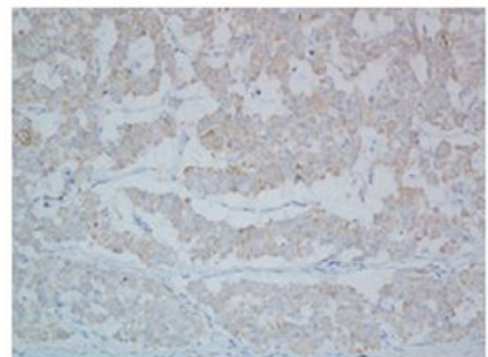

Anti-CK

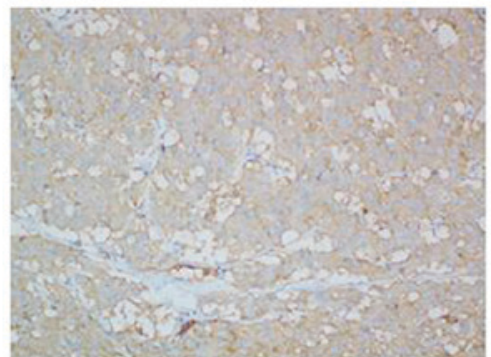

Anti-SYN

Figure 4. Immunohistochemical analysis of CgA, CK and SYN expression in tumor tissue (magnification, x200). Samples of tumor tissues were stained for the indicated genes to determine expression. A representative section is presented. CgA, chromagranin; CK, cytokeratin; SYN, synaptophysin.

was observed in 2 patients and was considered to be caused by pancreatic portal hypertension.
The differential diagnosis of NPNEC should allow differentiation from pancreatic cancer, cystadenoma or 
cystadenocarcinoma, solid pseudopapillary tumors and intraductal papillary mucinous neoplasms (7). Pancreatic cancer is characterized by tumors that lack blood supply. Tumor enhancement is usually not evident, the border between tumors and pancreatic tissue is unclear, and pancreatic cancer usually involves the pancreaticobiliary duct and causes dilation $(19,23)$. The dilated pancreatic duct typically presents beaded changes. The pancreas at the distal end of the tumor usually exhibits atrophy, and the peripancreatic structure and blood vessels are usually invaded (6). The cyst fluid density is relatively low and the thickness of the cyst wall is homogeneous or inhomogeneous. The cyst wall is sometimes combined with mural nodules and may exhibit separation, and enhanced scanning indicates mild enhancement of the cyst wall and fibrous separation (19). Furthermore, calcification may occur in the cyst wall and its contents (19).

Intraductal papillary mucinous neoplasms primarily occur in older men and $80 \%$ of tumors are located in the pancreatic head. These lesions mainly result in dilation of the branched pancreatic duct and/or the main pancreatic duct. Lobulated cystic lesions (mucous plugs), papillary nodules and separations inside are present $(18,22)$. The lesions are connected to the main pancreatic duct and severe atrophy of the pancreas is usually observed (18). A solid pseudopapillary tumor is a low-grade malignant tumor that usually occurs in middle-aged and young women, and presents as a solid-cystic mass. The solid regions are typically located at the surrounding areas of tumors and enhanced scanning usually presents gradual enhancement (19). The features of the aforementioned tumors may be used to differentiate them from NPNEC.

In conclusion, the present study demonstrated that the clinical manifestations of NPNEC do not have evident specificity. Patients often sought treatment late, the masses were large and there were certain characteristic changes on $\mathrm{CT}$ images. For patients with pancreatic tumors that have a large volume, an abundant blood supply, obvious enhancement, combined necrotic cystic lesions and infiltrative signs in the surrounding tissue, the possibility of NPNEC should be considered, despite it being a rare form of pancreatic carcinoma. The final diagnosis relies on pathological examination.

\section{Acknowledgements}

The current study was supported by a grant from Zhejiang Medical Technology and Education, Zhejiang, China (grant no. 2014KYB124) awarded to Dr Jibo Hu.

\section{References}

1. Burns WR and Edil BH: Neuroendocrine pancreatic tumors: Guidelines for management and update. Curr Treat Options Oncol 13: 24-34, 2012.

2. Shi LN, Qiu ZL, Wu CG and Luo QY: A rare case of calf muscle metastasis from a non-functional pancreatic neuroendocrine carcinoma. Iran J Radiol 12: e11637, 2015.

3. Metz DC and Jensen RT: Gastrointestinal neuroendocrine tumors: Pancreatic endocrine tumors. Gastroenterology 135 1469-1492, 2008.
4. Ehehalt F, Saeger HD, Schmidt CM and Grützmann R: Neuroendocrine tumors of the pancreas. Oncologist 14: 456-467, 2009.

5. Klöppel G: Classification and pathology of gastroenteropancreatic neuroendocrine neoplasms. Endocr Relat Cancer 18 (Suppl 1): S1-S16, 2011.

6. Lewis RB, Lattin GE Jr and Paal E: Pancreatic endocrine tumors: Radiologic-clinicopathologic correlation. Radiographics 30: 1445-1464, 2010

7. Bettini R, Boninsegna L, Mantovani W, Capelli P, Bassi C, Pederzoli P, Delle Fave GF, Panzuto F, Scarpa A and Falconi M: Prognostic factors at diagnosis and value of WHO classification in a mono-institutional series of 180 non-functioning pancreatic endocrine tumours. Ann Oncol 19: 903-908, 2008.

8. Kent RB III, van Heerden JA and Weiland LH: Nonfunctioning islet cell tumors. Ann Surg 193: 185-190, 1981.

9. Bartsch DK, Schilling T, Ramaswamy A, Gerdes B, Celik I, Wagner HJ, Simon B and Rothmund M: Management of nonfunctioning islet cell carcinomas. World J Surg 24: 1418-1424, 2000.

10. Solorzano CC, Lee JE, Pisters PW, Vauthey JN, Ayers GD, Jean ME, Gagel RF, Ajani JA, Wolff RA and Evans DB: Nonfunctioning islet cell carcinoma of the pancreas: Survival results in a contemporary series of 163 patients. Surgery 130: 1078-1085, 2001

11. Gullo L, Migliori M, Falconi M, Pederzoli P, Bettini R, Casadei R, Delle Fave G, Corleto VD, Ceccarelli C, Santini D and Tomassetti P: Nonfunctioning pancreatic endocrine tumors: A multicenter clinical study. Am J Gastroenterol 98: 2435-2439, 2003.

12. Falconi M, Plockinger U, Kwekkeboom DJ, Manfredi R, Korner M, Kvols L, Pape UF, Ricke J, Goretzki PE, Wildi S, et al: Well-differentiated pancreatic nonfunctioning tumors/carcinoma. Neuroendocrinology 84: 196-211, 2006.

13. Freelove R and Walling AD: Pancreatic cancer: Diagnosis and management. Am Fam Physician 73: 485-492, 2006.

14. Nakamura K, Nagata C, Wada K, Tamai Y, Tsuji M, Takatsuka N and Shimizu H: Cigarette smoking and other lifestyle factors in relation to the risk of pancreatic cancer death: A prospective cohort study in Japan. Jpn J Clin Oncol 41: 225-231, 2011.

15. Michaud DS, Giovannucci E, Willett WC, Colditz GA, Stampfer MJ and Fuchs CS: Physical activity, obesity, height, and the risk of pancreatic cancer. JAMA 286: 921-929, 2001.

16. Ryan DP, Hong TS and Bardeesy N: Pancreatic adenocarcinoma. N Engl J Med 371: 2140-2141, 2014.

17. Ehrhardt AR, Reuter S, Buck AK, Haenle MM, Mason RA, Gabelmann A, Kern P and Kratzer W: Assessment of disease activity in alveolar echinococcosis: A comparison of contrast enhanced ultrasound, three-phase helical CT and [(18)F] fluorodeoxyglucose positron emission tomography. Abdom Imaging 32: 730-736, 2007.

18. Franko J, Feng W, Yip L, Genovese E and Moser AJ: Non-functional neuroendocrine carcinoma of the pancreas: Incidence, tumor biology and outcomes in 2,158 patients. J Gastrointest Surg 14: 541-548, 2010.

19. Balci NC and Semelka RC: Radiologic features of cystic, endocrine and other pancreatic neoplasms. Eur J Radiol 38: 113-119, 2001.

20. Nakazawa S, Yoshino J, Nakamura T, Yamanaka T, Hase $S$, Kojima Y, Ohasi S and Niwa Y: Endoscopic ultrasonography of gastric myogenic tumor. A comparative study between histology and ultrasonography. J Ultrasound Med 8: 353-359, 1989.

21. Noone TC, Hosey J, Firat Z and Semelka RC: Imaging and localization of islet-cell tumours of the pancreas on CT and MRI. Best Pract Res Clin Endocrinol Metab 19: 195-211, 2005.

22. Debray MP, Geoffroy O, Laissy JP, Lebtahi R, Silbermann-Hoffman O, Henry-Feugeas MC, Cadiot G, Mignon M and Schouman-Claeys E: Imaging appearances of metastases from neuroendocrine tumours of the pancreas. Br J Radiol 74: 1065-1070, 2001

23. Gallotti A, Johnston RP, Bonaffini PA, Ingkakul T, Deshpande V, Fernández-del Castillo C and Sahani DV: Incidental neuroendocrine tumors of the pancreas: MDCT findings and features of malignancy. AJR Am J Roentgenol 200: 355-362, 2013. 